\title{
Generation of deterministic eddy structure of the flow as an analogue of the phase transition of the second kind. Development of ideas of academician I. I. Novikov
}

\author{
Olga Mitrofanova* \\ National Nuclear Research University "MEPHI", 115409, Kashirskoye sh. 31, Moscow, Russia
}

\begin{abstract}
The analysis of the physical processes leading to the structure formation of fluids has been done. The proposed theoretical approach can be used both for the neutral, conductive and magnetic fluids. In the present work the main attention is paid to the vortex and swirl flows of Newtonian fluids. Critical conditions accompanied the occurrence of hydrodynamic instabilities with a structural restructuring of flow field have been identified due to carried out physical experiments on generation of largescale steady-stable vortex structures in channels of complicated geometry. Examples of topological models of the internal structure of the flow under condition of the phase transition of the second kind without changing of the aggregation state of the environment have been considered. It is shown that for vortex and swirl flows the parameter of helicity associated with the emergence of the internal angular momentum of the fluid and the structuring of the flow depending on the intensity of the local vorticity and orientation of longitudinal helical vortices can be accepted as the order parameter.
\end{abstract}

Theoretical analysis indicates the overall thermodynamic nature of the crisis phenomena associated with the formation of ordered vortex structures and transition the system to a stable, energetically more favorable state. The thermodynamic approach developed by academician I.I. Novikov is based on the ideas of Gibbs about the stability boundaries and the critical state of matter. General character of the phenomenon "stability crisis" was examined by I.I. Novikov from the thermodynamic point of view in his monograph [1]. Notion critical phenomena in this case refers to different abnomal situations which occur during the movement of liquid or gas. For example: the crisis of flow around, secondary flows of different classes, the crisis of the expiry of the swirl flow (critical flow), the generation of deterministic eddy structure of the flow in the acoustic flows, etc. The phase transition of the second kind corresponds to the case of transition of a thermodynamic system in a continuous manner without decay through a state of zero stability, in which $(\partial P / \partial V)_{T}=0$, with the formation of new stable structures.

* Corresponding author: omitr@yandex.ru 
According to [1], the condition for thermodynamic equilibrium of the hydromechanical system is the minimum of the thermodynamic potential - the Gibbs energy $\Phi$ at constant pressure $\mathrm{P}$ and temperature $\mathrm{T}$. Under this condition, the hydromechanical system is stable against external impact or disturbance.

Given the fact that when the deterministic steady vortex structure of the flow is produced by a moving fluid under the action of mass forces (the nature of which may be varies), and the symmetry of a homogeneous medium is disturbed, in the expression for the thermodynamic potential the Gibbs energy $\Phi$ (1) and internal energy $U$ (2) must be entered the term associated with the order parameter (3). This term characterizes the degree of asymmetry of the environment due to the formation of an ordered structure.

$$
\begin{gathered}
\Phi=U-T S+p V-\zeta_{i} \eta_{i} \\
d U=T d S-p d V+\zeta_{i} d \eta_{i} \\
\zeta_{i}=\left(\partial U / \partial \eta_{i}\right)_{S, V}
\end{gathered}
$$

where $T$ - temperature, $P$ - pressure, $V$ - volume, $S$ - entropy, - $\zeta_{i}$ acting on the body due to external force interrelated with the order parameter $\eta_{i}$.

As an illustrative example of the critical restructuring of the flow in Fig.1 it is shown the instability of Taylor.

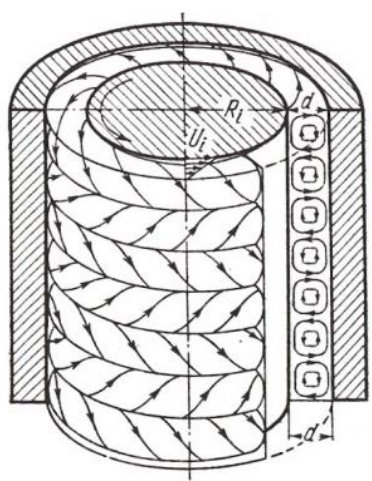

$\mathrm{a}$

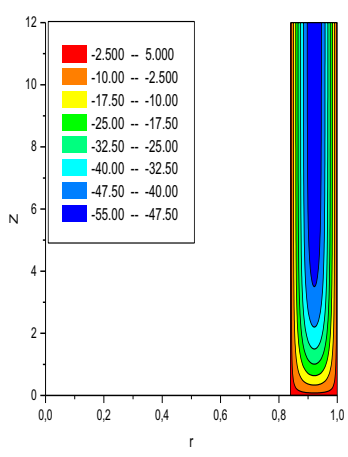

$\mathrm{b}$

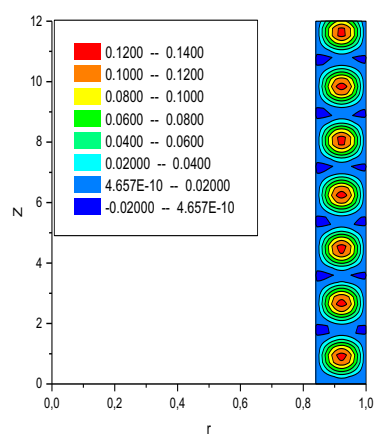

c

Fig. 1. The Taylor's vortices: a) the streamlined structure of flow in excess of the critical value of Taylor number, corresponding to the first singular point of the analytic solution $\mu_{1}=19,65$ [3]; b), c) - distributions of tangential velocity in dependence on dimensionless values of the intensity of the flow vorticity $\mathrm{K}$ near a singular point : b) $\mathrm{K}=19,64\left(K \rightarrow \mu_{1-}\right)$, c) $\mathrm{K}=19,66\left(K \rightarrow \mu_{1+}\right)$.

The phenomenon of the crisis of hydrodynamic stability in this case is connected with a sharp restructuring of the flow pattern and the formation of a system of toroidal vortices (Fig. $1 \mathrm{a}, \mathrm{c}$ ). Thus, as shows the theoretical analysis and comparison with experimental results, the main volume of channel containes non-dissipative vortex structures, obeying the law of a screw flow (Fig. 1 a), and the effect of viscosity is shifted in a narrow region on the boundary of the vortices. 
Using the proposed in [1] a thermodynamic approach, which considers the analogy between the phase transition of the second kind and the occurrence of instability, makes it possible to identify the universalism of the development of instabilities typical for nonequilibrium dissipative structures transitions to a new, energetically more favorable forms under changing external conditions.

To identify the most significant physical laws that lead to structural stability of doubly periodic vortex motions with two basic frequencies $\omega_{1}$ and $\omega_{2}$, the vortex structures represented in Fig. 2 a, b can be considered as model objects.

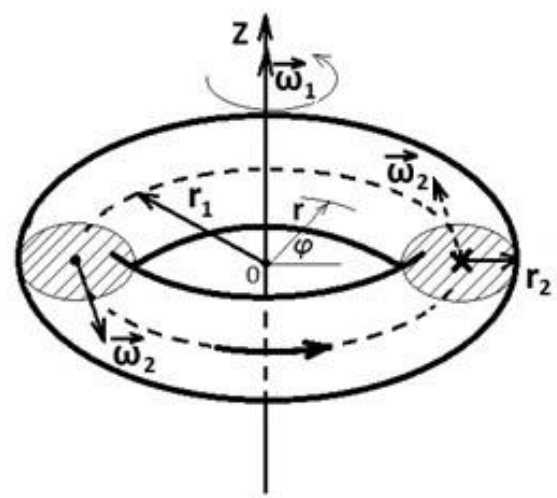

a)

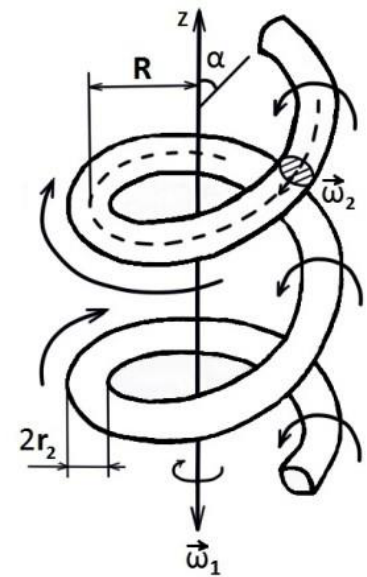

b)

Fig. 2. Doubly periodic vortex motion: a) model of a toroidal vortex with double rotation; b ) screwhelix structure of the vortex, in this case, the radius $\mathrm{rl}$ corresponds to the radius of curvature of the helix, the twist angle of which around its longitudinal axis z equal to: $r_{1}=R / \sin ^{2} \alpha$.

Since the choice of the order parameter, which can be scalar, vector, and tensor quantity, not limited, it is advisable to use the parameter of helicity $\mathrm{H}$ [3] as the order parameter at helical-vortex motion of the fluid:

$$
H=\mathbf{u} \cdot \boldsymbol{\omega} \neq 0,
$$

where $\mathbf{u}$ is the local flow velocity, $\boldsymbol{\omega}=\operatorname{rot} \mathbf{u}$ is a vector of vorticity at a given point of the flow.

The condition $\vec{u} \cdot \vec{\omega} \neq 0$ corresponding to appearance of helicity is directly conformed with kinematic condition of a screw motion by Gromeka [4]:

$$
\operatorname{rot} \vec{u}=k \vec{u}
$$

where $k$ is the vorticity intensity characterizing the ratio of circulation $u_{\phi} r$ to the stream function (flow rate) $\psi=u_{z} r^{2} / 2$. Physically, this means that the vortex lines are helical lines and coincide with the mechanical trajectories of liquid particles motion [3].

To assess the degree of ordering of the liquid medium during the formation of vortical structures, represented in Fig. 2 a, b, we can obtain the following estimation of the magnitude of helicity in the region of spatial localization of the vortex: 


$$
H=|\mathbf{u}| \cdot|\boldsymbol{\omega}|=\sqrt{u_{1}^{2}+u_{2}^{2}} \cdot \sqrt{\omega_{1}^{2}+\omega_{2}^{2}}=\frac{1}{\sqrt{r_{1} r_{2}}}\left(\omega_{1}^{2} r_{1}^{2}+\omega_{2}^{2} r_{2}^{2}\right)=\frac{|\mathbf{u}|}{k},
$$

$u_{1}, u_{2}$ - components of linear velocity of a screw motion, $|\mathbf{u}|=\sqrt{u_{1}^{2}+u_{2}^{2}}$.

According to the theory of self-organized criticality proposed in [5], in non-equilibrium systems can be the processes of self-organization. In addition from the set describing the system variables, you can allocate a small number of control parameters (order parameters) the leading variables that adjusts all other. A characteristic feature of such systems is scale invariance - lack for events or objects their own characteristic scales (sizes, durations, energies, etc.).

Theoretical analysis shows that in this case restructuring the vortex flow structure occurs when changing the intensity of vorticity, which for the considered models (Fig. 2 a, b) is estimated as $k=\frac{1}{\sqrt{r_{1} r_{2}}}$.

In the mathematical solution given in [3] that describes the instability of Taylor (Fig. 1), the existence of bifurcation points with defined values of the parameter $k$, which may correspond to different values of the radii $r_{1}$ and $r_{2}$, indicates scale invariance of the vortex formation [5].

Introduction in consideration of the order parameter gives the possibility to apply the formalism of thermodynamic analysis of phase transitions of the second kind for liquid under condition the formation of stable vortex structure of the flow moving in the field of mass forces.

This work was supported by RFBR grant 16-08-00687 and the program of increasing the competitive ability of National Research Nuclear University MEPhI (agreement with the Ministry of Education and Science of the Russian Federation of August 27, 2013, project no. 02.a03.21.0005).

\section{References}

1. I.I. Novikov Thermodynamics of spinodals and phase transitions (2000) [in Russian]

2. L.D. Landau, E.M. Lifshits Theoretical physics: a tutorial. V. Statistical physics. Part I. - 5-e Izd., (2005)

3. O.V. Mitrofanova Hydrodynamics and heat transfer of swirl flows in channels of nuclear power installations (2010) [in Russian

4. I.S. Gromeka, Complete works, Ac. Sci. 3, 107 (1952)

5. P. Bak, C. Tang, K. Wiesenfeld, Self-organized criticality: An explanation of the 1/f noise Phys. Rev. Lett. 59, 381 (1987) 